\title{
Fotografías de obras de Zurbarán o atribucuines, conservadas en el Archivo de Ruiz Vernacci
}

\author{
Ana María Gutiérrez Martínez \\ Conservadora Archivo Ruiz Vernacci \\ Instituto del Patrimonio Histórico Español \\ El Archivo Ruiz Vernacci agrupa en sus fondos negativos \\ fotográficos que a lo largo de un siglo han realizado los \\ distintos fotógrafos propietarios del establecimiento \\ comercial conocido bajo diferentes nombres comerciales \\ situado en Madrid, y que ha sido utilizado ampliamente \\ por Investigadores del Arte y Arquitectos. En la actualidad \\ depende del Instituto del Patrimonio Histórico Español \\ ( $M^{\circ}$ de Educación y Cultura)!
}

En los esquemas que figuran a continuación hemos recogido una relación de los negativos originales del archivo, de las obras del propio Zurbarán, de obras atribuidas a él o de copias, realizados a lo largo de la segunda mitad del S. XIX y seis décadas del presente siglo.

Estos negativos permitieron, a lo largo del tiempo, obtener multitud de copias positivas en papel, de diferentes formatos, que dieron a conocer la obra del pintor. El primero en fotografiar y comercializar las reproducciones de sus pinturas fue J. Laurent, que ya en 1863 había fotografiado La Virgen, S. José y el Niño, La Aparición de S. Pedro Apóstol a S. Pedro Nolasco y La Visión de S. Pedro Nolasco en la Colección del Museo Real.

En 1867 había añadido a sus catálogos de fotografías a la venta otros dos nuevos cuadros atribuidos entonces a Zurbarán Retrato del poeta español Luis de Góngora² y El niño Jesús dormido.

En 1868 aparece un Suplemento al Catálogo y anuncia fotografías de cinco cuadros de Monjes mercedarios, Un jesuita en éxtasis y S. Francisco difunto.

En 1872, suma a las fotografías anteriores seis de la Galería del Duque de Montpensier en su Palacio de S. Telmo en Sevilla ${ }^{3}$, nueve del Hospital de la Sangre ${ }^{4}$, y catorce del Museo Provincial de Sevilla.

En 1874 establece una Sociedad Regular Colectiva con, Catalina Melina Dosch y D. Manuel Sánchez Rubio, por lo que seguramente inscribe las fotografías como suyas en el Registro de la Propiedad Intelectual de Madrid en Septiembre de 1875.
J. Laurent constituye Sociedad Regular Colectiva en 1878 con Catalina Melina Dosch, pasando a denominarse J. Laurent y Cia., y un año después ya habían fotografiado once cuadros del Museo Provincial de Cádiz y dos más, uno de la Catedral de Sevilla y otro de la de Palencia.

El día | de julio de |88| Laurent cede su participación en la sociedad a su hijastra Catalina Melina Dosch5.

J. Lacoste y Borde, adquiere los fondos de la Casa Laurent y la anuncia como "Antigua Casa Laurent" en los primeros años de este siglo y fotografía fundamentalmente los cuadros existentes en el Museo del Prado, ya que fue su fotógrafo oficial, y los de las Colecciones Lázaro, Marqués de Casa Torres y algunas otras.

El 12 de agosto de 1902 se forma la Sociedad Casa Laurent. Sucesor J. Lacoste y Compañía, para la explotación de la fotografía y la fototipia y con aportación de 100.000 pts como capital social aportado entre los dos socios: José Lacoste y Borde, Fotógrafo (director de la sección comercial) y Angel Rodríguez de Zúñiga, abogado (director de la sección técnica). En Febrero de 1904 se disuelve la Sociedad, quedando al frente de la misma el propio Lacoste y su esposa Da Margarita Amengual y Perelló.

El último de los fotógrafos del Archivo, la firma comercial Ruiz Vernacci, realiza fotografías, por encargo, de cuadros que figuraron en exposiciones de Zurbarán y en colecciones privadas.

Aunque en el Archivo no hay demasiados negativos datados originariamente, sus imágenes nos permiten conocer el estado de las pinturas en cada momento y quienes eran sus propietarios, ambos datos de gran importancia para el estudio de las obras.

Se han incluido en la relación de los negativos los números con que figuran en el Catálogo de Gudiol, el $n^{\circ}$ que tenían en el Museo o Colección donde formaban parte de sus fondos, el título por el que se conocía la obra, el formato del negativo, el $n^{\circ}$ de Inventario Antiguo asignado por los fotógrafos del Archivo, el $N^{\circ}$ de Inventario Moderno por el que se encuentran Catalogadas en el Archivo, el $n^{\circ}$ de reserva del negativo, la medida que Gudiol ó Pemán dan de la pintura, la medida real que tenían en el momento de ser fotografiadas, los años en que aparecen publicadas en los Catálogos editados por Laurent, y la procedencia y dis- 
tintas colecciones a que han pertenecido. En algunos casos aparecen dos números de Inventario moderno relacionados con el mismo número de Inventario Antiguo, lo que significa que existen dos negativos, dado que el fotógrafo acostumbraba a tomar más de un negativo, dado la fragilidad del soporte de cristal.

Una gran parte de las obras de Zurbarán fueron desamortizadas de los Conventos y Monasterios de Sevilla: Convento de la Merced Descalza, Cartuja de Ntra. Sra. de las Cuevas, Convento Grande de la Orden Mercedaria, Convento dominico de Sto. Tomás, Santo Domingo de Porta Coeli, Convento de los Capuchinos, la Casa Profesa de la Compañía de Jesús.
Los que constituían el retablo de la Cartuja de Ntra. Sra. de la Defensión de Jerez de la Frontera, formaron parte de la Galería Española de Louis Felipe en Louvre en 1838, y cuya venta fue discutida en las Cortes. Las siete tablas de los padres cartujos del pasadizo del Sagrario de la Cartuja de Jerez y los dos ángeles turiferarios, hoy en el Museo de Bellas Artes de Cádiz.

Dar a conocer la existencia de reproducciones fotográficas de los cuadros en distintas épocas, nos parece trascendental, a la hora de emprender el estudio, documentación, investigación y restauración de cada obra, por lo cual damos a conocer a través de estos esquemas información de su existencia a los investigadores interesados.

\section{CUADROS DE ZURBARÁN, 0 ATRIBUIDOS A ÉL, FOTOGRAFIADOS POR LAURENT Y CIA. 6 SIGUIENTES FOTÓGRAFOS ${ }^{7}$ DEL ARCHIVO}

\section{CAT. CUADROS DE LA CARTUJA DE JEREZ EN EL MUSEO DE CÁDIZ}

\begin{tabular}{|c|c|c|c|c|c|c|c|c|c|c|c|c|}
\hline $\begin{array}{l}\text { Cat.M }{ }^{0} \\
1835\end{array}$ & $\begin{array}{l}\text { Cat. } \\
\text { Gudiol }\end{array}$ & Título obra & $\begin{array}{l}\text { Formato } \\
\text { placa }\end{array}$ & N.I.A. & N.I.M & $\begin{array}{l}\text { Reser } \\
\mathbf{v}\end{array}$ & $\begin{array}{l}\mathrm{N}^{\circ} \\
\text { Inso } \\
\text { rit }\end{array}$ & $\begin{array}{l}\text { MEDIDA } \\
\text { actual tabla }\end{array}$ & $\begin{array}{l}\text { MEDIDA } \\
\text { Pemán } \\
\text { Catálogo } \\
\text { Museo. }\end{array}$ & $\begin{array}{l}\text { Med mareo } \\
\text { fotog } \\
\text { Laurent } \\
\text { Arr } \times \text { Dcha } \\
\text { Ab x Izd }\end{array}$ & $\begin{array}{l}\text { Año } \\
\text { Catálog } \\
0 \\
\text { Laurent. }\end{array}$ & Procedencia de las obras \\
\hline 68 & 141 & $\begin{array}{l}\text { S. Hugo obispo de } \\
\text { Lincoln }\end{array}$ & $27 \times 36$ & $A-1820$ & 8876 & $R-615$ & 90 & $122.2 \times 63.7$ & $120.7 \times 62.6$ & $\begin{array}{l}18.4 \times 32.8 \mathrm{i} \\
18 \times 33.3 \mathrm{i}\end{array}$ & $1879^{8}$ & $\begin{array}{l}\text { Carj. Jerez-Pasadizo del } \\
\text { Sagrario, segundo lugar } \\
\text { epistola }\end{array}$ \\
\hline 69 & 138 & S. Artholdo & $\begin{array}{l}27 \times 36 \\
18 \times 24\end{array}$ & A-1821 & $\begin{array}{l}4576 \\
27.4 .17\end{array}$ & R-613 & 88 & $122,2 \times 63,7$ & $\begin{array}{l}120,7 \times 62,6 \\
\text { Cat.1964. } \\
\text { Fot RV }\end{array}$ & $\begin{array}{l}18,5 \times 32,7 \mathrm{i} \\
18,0 \times 33,6 \mathrm{i}\end{array}$ & 1879 & $\begin{array}{l}\text { Carj. Jerez-Pasadizo del } \\
\text { Sagrario, lado de la } \\
\text { cpistola }\end{array}$ \\
\hline 72 & 136 & S. Bruno & $\begin{array}{l}27 \times 36 \\
18 \times 24\end{array}$ & $\begin{array}{l}\text { A-1823 } \\
\text { A-1823. }\end{array}$ & $\begin{array}{l}3854 \\
27.4 .12\end{array}$ & R-614 & 89 & $122,2 \times 65,5$ & $\begin{array}{l}120,7 \times 62,6 \\
\text { Cat. } 1964 . \\
\text { Fot RV }\end{array}$ & $\begin{array}{l}18,5 \times 32,0 \mathrm{i} \\
18,0 \times 31,8 \mathrm{i}\end{array}$ & 1879 & $\begin{array}{l}\text { Carj. Jerez-Pasadizo del } \\
\text { Sagrario, primer lugar } \\
\text { evangelio }\end{array}$ \\
\hline 73 & 142 & $\begin{array}{l}\text { Beato John } \\
\text { Huoughton, [con } \\
\text { mascara] }\end{array}$ & $\begin{array}{l}27 \times 36 \\
18 \times 24\end{array}$ & $\begin{array}{l}\text { A-1824 } \\
\text { A-1824 }\end{array}$ & $\begin{array}{l}3847 \\
27.4 .14\end{array}$ & & & $122,4 \times 65,6$ & $\begin{array}{l}120,7 \times 62,6 \\
\text { Cat.1964. } \\
\text { Fot RV }\end{array}$ & $\begin{array}{l}18,3 \times 32 \mathrm{i} \\
18,4 \times 32,5 \mathrm{i}\end{array}$ & 1879 & $\begin{array}{l}\text { Carj. Jerez-Pasadizo del } \\
\text { Sagrario, primer lugar } \\
\text { epistola }\end{array}$ \\
\hline 75 & 134 & $\begin{array}{l}\text { Angel turiferario } \\
\text { (hacia Dch) }\end{array}$ & $\begin{array}{r}27 \times 36 \\
27 \times 36 \\
\\
18 \times 24\end{array}$ & $\begin{array}{l}\text { A-1825 } \\
\text { A-1 } 1825 \\
\text { A-1825 }\end{array}$ & $\begin{array}{l}2231 \\
9031 \\
\\
27.4 .84\end{array}$ & $\mathrm{R}-611$ & 86 & $122,4 \times 65,6$ & $\begin{array}{l}\text { I20,7×62,6 } \\
\text { Cat.1964. } \\
\text { Fot RV }\end{array}$ & $\begin{array}{l}18,4 \times 32 \\
17,8 \times 31,5\end{array}$ & 1879 & $\begin{array}{l}\text { Carj. Jerez- ¿ interior del } \\
\text { pasillo del Sagrario, } \\
\text { Después portezuela del } \\
\text { pasillo, lado evangelio }\end{array}$ \\
\hline 76 & 135 & $\begin{array}{l}\text { Angel turiferario } \\
\text { (hacia lzq) }\end{array}$ & $\begin{array}{l}27 \times 36 \\
18 \times 24\end{array}$ & $\begin{array}{l}\text { A-1826 } \\
\text { A-1826 }\end{array}$ & $\begin{array}{l}3586 \\
\\
27.4 .16\end{array}$ & $R-608$ & 83 & $122,3 \times 65,4$ & $\begin{array}{l}\text { I } 20,7 \times 62,6 \\
\text { Cat.1964. } \\
\text { Fot RV }\end{array}$ & $\begin{array}{l}19,1 \times 2,9 \mathrm{i} \\
18,55 \times 33,1 \\
\mathrm{i}\end{array}$ & 1879 & $\begin{array}{l}\text { Carj. Jerez-i interior del } \\
\text { pasillo del Sagrario, } \\
\text { Después portezuela del } \\
\text { pasillo, lado epístola }\end{array}$ \\
\hline [74] & 140 & $\begin{array}{l}\text { Cardenal Nicolaus } \\
\text { Albergati }\end{array}$ & $\begin{array}{l}27 \times 36 \\
18 \times 24\end{array}$ & A-1827 & $\begin{array}{l}120 \\
27.1 .145\end{array}$ & $R-609$ & 84 & $122,2 \times 63,4$ & $\begin{array}{l}120,7 \times 62,6 \\
\text { Cat.1964. } \\
\text { Fot RV }\end{array}$ & $\begin{array}{l}17,8 \times 32,7 \mathrm{i} \\
18,1 \times 33,2 \mathrm{i}\end{array}$ & 1879 & $\begin{array}{l}\text { Carj. Jerez-Pasadizo del } \\
\text { Sagrario, lado de la } \\
\text { epistola }\end{array}$ \\
\hline$[70]$ & 137 & $\begin{array}{l}\text { S. Anthelmo, } \\
\text { obispo de Belley }\end{array}$ & $\begin{array}{l}27 \times 36 \\
18 \times 24\end{array}$ & $\begin{array}{l}\text { A- } 1828 \\
\text { A- } 1828\end{array}$ & $\begin{array}{l}3860 \\
27.4 .13\end{array}$ & $R-616$ & 91 & $122,5 \times 63,5$ & $\begin{array}{l}120,7 \times 62,6 \\
\text { Cat.1964. } \\
\text { Fot RV }\end{array}$ & $\begin{array}{l}18 \times 32,9 \mathrm{i} \\
17,55 \times 33 \mathrm{i}\end{array}$ & 1879 & $\begin{array}{l}\text { Carj. Jerez-Pasadizo del } \\
\text { Sagrario, tercero o cuarto } \\
\text { lugar evangelio }\end{array}$ \\
\hline [71] & 139 & $\begin{array}{l}\text { S. Hugo, obispo de } \\
\text { Grenoble }\end{array}$ & $\begin{array}{l}27 \times 36 \\
18 \times 24\end{array}$ & $\begin{array}{l}\text { A-1830 } \\
\text { A- } 1830\end{array}$ & $\begin{array}{l}118 \\
27.1 .146\end{array}$ & $\mathrm{R}-610$ & .85 & $122,8 \times 63,5$ & $\begin{array}{l}120,7 \times 62,6 \\
\text { Cat.1964. } \\
\text { Fot RV }\end{array}$ & $\begin{array}{l}18,7 \times 33,2 \mathrm{i} \\
18 \times 33,7 \mathrm{i}\end{array}$ & 1879 & $\begin{array}{l}\text { Carj. Jerez-Pasadizo del } \\
\text { Sagrario, segundo lugar } \\
\text { evangelio }\end{array}$ \\
\hline & $s / n$ & S. Amaldo & & & & & & & & & & \\
\hline
\end{tabular}




\section{GALERÍA DEL DUQUE DE MONTPENSIER, PALACIO DE S. TELMO. SEVILLA}

\begin{tabular}{|c|c|c|c|c|c|c|c|c|c|}
\hline $\begin{array}{l}\text { Cat.Col } \\
\text { Duque }\end{array}$ & $\begin{array}{l}\text { Formato } \\
\text { negativo. }\end{array}$ & $\begin{array}{l}\text { Catál } \\
\text { GUDIOL }\end{array}$ & N.I.A & N.I.M & Título obra & $\begin{array}{l}\mathrm{N}^{\circ} \\
\text { inscrito }\end{array}$ & $\begin{array}{l}\text { Med. Cat. } \\
\text { Laurent }\end{array}$ & $\begin{array}{l}\text { Año catá } \\
\text { Laurent }\end{array}$ & Procedencia de las obras \\
\hline 190 & $\begin{array}{l}27 \times 36 \\
27 \times 36\end{array}$ & 320 & $\begin{array}{l}A-1004^{10} \\
A-1004\end{array}$ & $\begin{array}{l}8444 \\
8751\end{array}$ & $\begin{array}{l}\text { La Vièrge de la Merci avec } \\
\text { divers Saints }\end{array}$ & $\begin{array}{l}\text { R-354 } \\
\text { T5593 }\end{array}$ & $\begin{array}{l}1,68 \times 1,26 \\
\text { con escala }\end{array}$ & $\begin{array}{l}1872 \\
1879\end{array}$ & $\begin{array}{l}\text { [Sev:Convento Grande Merced Descalza } \\
\text { Paris: Col. Louis Felipe, } 1838 \\
\text { Londres: venta Christie's, } 1853 \\
\text { Sev. Duque de Montpensier. Catal: } 1866 \\
\text { Château de Randan (Auverne): Condesa de París } \\
\text { Madrid. Duque de Montpensier } \\
\text { Marqués de Valdeterrazo] }\end{array}$ \\
\hline 186 & {$[27 \times 36]^{11}$} & 122 & A-1005 & & L'Annonciation & & $2,66 \times 1,82$ & $\begin{array}{l}1872 \\
1879\end{array}$ & $\begin{array}{l}\text { [Jerez: Cartuja de Nitra Sra. Defensión } \\
\text { Paris: Louvre Galeria Espatiola de Louis Felipe, } 1838 \text { n }^{\circ} \\
325 \\
\text { Londres: Venta Christie's, } 1853 \text { n }^{\circ} 157 \\
\text { Sev. Duque de Montpensier. Catal: } 1866 \mathrm{n}^{\circ} 186 \\
\text { General de Beylie (1904) } \\
\text { Grenoble: M. B.A. no } 559 \text { ] }\end{array}$ \\
\hline 179 & {$[27 \times 36]$} & 123 & A-1006 & & L'Adoration des bergers & & $2,66 \times 1,82$ & $\begin{array}{l}1872 \\
1879\end{array}$ & $\begin{array}{l}\text { [Jerez: Cartuja de Ntra Sra. Defensión } \\
\text { Paris: Louvre Galeria Española de Louis Felipe, } 1838 \mathrm{n}^{\circ} \\
327 \\
\text { Londres: venta Christie's, } 1853 \mathrm{n}^{\circ} 159 \\
\text { Sev. Duque de Montpensier. Catal: } 1866 \mathrm{n}^{\circ} 179 \\
\text { General de Beylie (1904) } \\
\text { Grenoble: M. B.A. } \mathrm{n}^{\circ} \text { 560] }\end{array}$ \\
\hline 189 & $27 \times 36$ & 125 & $\begin{array}{l}\text { A-1007 } \\
\text { A-1007 }\end{array}$ & $\begin{array}{l}9347 \\
532\end{array}$ & L'Adoratión dos rois máges & $\mathrm{J}-460$ & $2,66 \times 1,82$ & $\begin{array}{l}1868 \\
1872 \\
1879\end{array}$ & $\begin{array}{l}\text { [Jerez: Cartuja de Nta Sra. Defensión } \\
\text { Paris: Louvre Galeria Española de Louis Felipe, } 1838 \text { n }^{\circ} \\
328 \\
\text { Londres: venta Cluristie's, } 1853 \\
\text { Sev. Duque de Montpensier. Catal: } 1866 \\
\text { General de Beylie (1904) } \\
\text { Grenoble: M. B.A. no } 561 \text { ] }\end{array}$ \\
\hline 174 & {$[27 \times 36]$} & 124 & A-1008 & & La Circoncision & & $2,66 \times 1,82$ & $\begin{array}{l}1872 \\
1879\end{array}$ & $\begin{array}{l}\text { [Jerez: Cartuja de Ntra Sra. Defensión } \\
\text { Paris: Louve Galeria Española de Louis Felipe, } 1838 \mathrm{n}^{\circ} \\
328 \\
\text { Londres: venta Christie's, } 1853 \mathrm{n}^{\circ} 140 \\
\text { Sev. Duque de Montpensier. Catal: } 1866, \mathrm{n}^{\circ} 174 \\
\text { General de Beylie (1904) } \\
\text { Grenoble: M. B.A no } \text { n }^{\circ} \text { ] }\end{array}$ \\
\hline 185 & $\begin{array}{l}27 \times 36 \\
27 \times 36 \\
\end{array}$ & $\begin{array}{l}\text { no } \\
\text { aparece }\end{array}$ & $\begin{array}{l}\text { A-1009 } \\
\text { A-1009 } \\
\end{array}$ & $\begin{array}{l}8777 \\
9414 \\
\end{array}$ & $\begin{array}{l}\text { Un moine (con papel en la } \\
\text { mano, sentado medio } \\
\text { cuerpo }\end{array}$ & $\begin{array}{l}\mathrm{R}-358 \\
\mathrm{~T} 591 \\
\mathrm{~J}-431 \\
\end{array}$ & $\begin{array}{l}1,05 \times 0,84 \\
\text { con escala }\end{array}$ & $\begin{array}{l}1872 \\
1879\end{array}$ & \\
\hline
\end{tabular}

HOSPITAL DE LA SANGRE, SEVILLA

\begin{tabular}{|c|c|c|c|c|c|c|c|c|}
\hline $\begin{array}{l}\text { ormat } \\
\text { ncg. }\end{array}$ & $\begin{array}{l}\text { Catál } \\
\text { GUDIOL }\end{array}$ & $\begin{array}{l}\text { GUDIOL } \\
\text { medida }\end{array}$ & N.I.A & N.I.M & Título obra & $\begin{array}{l}\text { Med.Cat. } \\
\text { Laurent }\end{array}$ & $\begin{array}{l}\text { Año } \\
\text { catál }\end{array}$ & Procedencia de las obras \\
\hline $\begin{array}{l}7 \times 36 \\
7 \times 36\end{array}$ & 428 & $181 \times 102$ & $\begin{array}{l}A-1055 \\
A-1055\end{array}$ & $\begin{array}{l}9463 \\
4163\end{array}$ & $\begin{array}{l}\text { La Vierge et l'enfant Jésus [V. del } \\
\text { Rosario] }\end{array}$ & $1,77 \times 1,02$ & $\begin{array}{l}1872 \\
1879\end{array}$ & $\begin{array}{l}\text { [Hospital de la Sangre en Sevilla } \\
M^{\circ} \text { Prov. B.A. Sevilla (1915)] }\end{array}$ \\
\hline $\begin{array}{l}7 \times 36 \\
7 \times 36\end{array}$ & 423 & $173 \times 102$ & $\begin{array}{l}\text { A-1056 } \\
\text { A-1056 }\end{array}$ & $\begin{array}{l}9395 \\
4173\end{array}$ & Sainte Eulalie & $1,69 \times 1,00$ & $\begin{array}{l}1872 \\
1879\end{array}$ & $\begin{array}{l}\text { [Hospital de la Sangre en Sevilla } \\
\text { Mo Prov. B.A. Sevilla (1915)] }\end{array}$ \\
\hline $\begin{array}{l}7 \times 36 \\
7 \times 36\end{array}$ & 430 & $169 \times 102$ & $\begin{array}{l}A-1057 \\
A-1057\end{array}$ & $\begin{array}{l}9403 \\
4169\end{array}$ & Sainte Catherine & $1,69 \times 1,00$ & $\begin{array}{l}1872 \\
1879\end{array}$ & $\begin{array}{l}\text { [Hospital de la Sangre en Sevilla } \\
\text { Mo Prov. B.A. Sevilla (1915)] }\end{array}$ \\
\hline $7 \times 36$ & 426 & $173 \times 102$ & A-1058 & 9454 & Sainte Engràce & $1,69 \times 1,00$ & $\begin{array}{l}1872 \\
1879\end{array}$ & $\begin{array}{l}\text { [Hospital de la Sangre en Sevilla } \\
\text { Mo Prov. B.A. Sevilla (1915)] }\end{array}$ \\
\hline $7 \times 36$ & 424 & $173 \times 103$ & A-1059 & 7808 & Sainte Barbre & $\begin{array}{l}1,69 \times 1,00 \\
\text { escal,cab, } \\
\text { exterior }\end{array}$ & $\begin{array}{l}1872 \\
1879\end{array}$ & $\begin{array}{l}\text { [Hospital de la Sangre en Sevilla } \\
\text { M}^{0} \text { Prov. B.A. Sevilla (1915)] }\end{array}$ \\
\hline $7 \times 36$ & 429 & $173 \times 102$ & A-1060 & 7817 & Sainte Mathilde & $1,69 \times 1,00$ & $\begin{array}{l}1872 \\
1879\end{array}$ & $\begin{array}{l}\text { [Hospital de la Sangre en Sevilla } \\
\text { Mo Prov. B.A. Sevilla (1915)] }\end{array}$ \\
\hline $\begin{array}{l}7 \times 36 \\
7 \times 36\end{array}$ & 425 & $171 \times 101$ & $\begin{array}{l}A-1061 \\
A-1061\end{array}$ & $\begin{array}{l}8515 \\
4166\end{array}$ & Sainte Agnès & $1,69 \mathrm{xl}, 00$ & $\begin{array}{l}1872 \\
1879\end{array}$ & $\begin{array}{l}\text { [Hospital de la Sangre en Sevilla } \\
\text { M}^{0} \text { Prov. B.A. Sevilla (1915)] }\end{array}$ \\
\hline $7 \times 36$ & 422 & $170 \times 101$ & A-1062 & 8135 & $\begin{array}{l}\text { Sainte Mathilde. } \\
\text { [Sta. Marina] }\end{array}$ & $1,69 \times 1,00$ & $\begin{array}{l}1872 \\
1879\end{array}$ & $\begin{array}{l}\text { [Hospital de la Sangre en Sevilla } \\
\text { M}^{0} \text { Prov. B.A. Sevilla (1915)] } \\
\text { [réplica en el Konst Museum de } \\
\text { Gotemburgo, fue de la Col. Louis Felipe] }\end{array}$ \\
\hline $7 \times 36$ & 427 & sin med & A-1063 & 8134 & Sainte Dorothée & $1,69 \times 1,00$ & $\begin{array}{l}1872 \\
1879\end{array}$ & $\begin{array}{l}\text { [Hospital de la Sangre en Sevilla } \\
\text { M}^{0} \text { Prov. B.A. Sevilla (1915)] }\end{array}$ \\
\hline
\end{tabular}




\section{MUSEO PROVINCIAL ${ }^{12}$, SEVILLA}






\begin{tabular}{|c|c|c|c|c|c|c|c|c|c|c|}
\hline \multicolumn{11}{|c|}{ MUSEO REAL/ MUSEO NACIONAL/ MUSEO DEL PRADO } \\
\hline $\begin{array}{l}\text { Cat } \\
\text { GUDioL }\end{array}$ & $\begin{array}{l}\text { GUDIOL } \\
\text { medida }\end{array}$ & $\begin{array}{l}\text { Tamaño } \\
\text { negativo }\end{array}$ & N.I.A & N.LM & Titulo obra & $\begin{array}{l}\mathrm{N}^{\circ} \\
\text { inscrit }\end{array}$ & $\begin{array}{l}\text { MedCat } \\
\text { Laurrent }\end{array}$ & $\begin{array}{l}\text { Año } \\
\text { catál }\end{array}$ & $\begin{array}{l}\text { No en el } \\
\text { Museo } \\
\text { Real }\end{array}$ & $\begin{array}{l}\text { No en el } \\
\text { Museo del } \\
\text { Prado }\end{array}$ \\
\hline & & & 67 & & Virgen, Sea José, Niño & & & 1863 & 67 & \\
\hline & & $\begin{array}{l}30 \times 40 \\
18 \times 24 \\
18 \times 24\end{array}$ & $\begin{array}{l}\text { A-216 } \\
54650^{14} \\
54650\end{array}$ & $\begin{array}{l}27.3 .138 \\
31.3 .95\end{array}$ & Retrato de Góngora ${ }^{15}$ & & $0,59 \times 0,46$ & $\begin{array}{l}1867 \\
1872 \\
1879\end{array}$ & 527 & 1085 \\
\hline 11 & $179 \times 223$ & $\begin{array}{l}27 \times 36 \\
30 \times 40 \\
18 \times 24\end{array}$ & $\begin{array}{l}165 \\
\text { A-217 } \\
{[A-13]} \\
\text { MP-1236 }\end{array}$ & 2022 & Visión S. Pierre Nolasque & $A-6$ & $1,80 \times 2,24$ & $\begin{array}{l}1863 \\
1867 \\
1872 \\
1879\end{array}$ & 190 & 1120 \\
\hline \multirow[t]{3}{*}{12} & $179 \times 223$ & $\begin{array}{l}30 \times 40 \\
18 \times 24\end{array}$ & $\begin{array}{l}164 \\
A-218 \\
M P-1237\end{array}$ & $\begin{array}{l}8.2 \\
25.2 .106\end{array}$ & $\begin{array}{l}\text { Apparition de Saint Pierre } \\
\text { Apôtre à Saint Pierre Nolasque }\end{array}$ & A-7 & $1,80 \times 2,24$ & $\begin{array}{l}1863 \\
1867 \\
1872 \\
1879\end{array}$ & 40 & 1121 \\
\hline & & $27 \times 36$ & $A-219$ & 1792 & L'enfant Jésus endormi & & $0,75 \times 1,00$ & $\begin{array}{l}1867 \\
1872 \\
1879\end{array}$ & 317 & 1133 \\
\hline & & $\begin{array}{l}27 \times 36 \\
27 \times 36 \\
18 \times 24\end{array}$ & $\begin{array}{l}\text { A-528 } \\
\text { A-528 } \\
\text { MP-1238 }\end{array}$ & $\begin{array}{l}1208 \\
5058 \\
25.2 .107\end{array}$ & Saint Francois défunt & Is543 & $2,73 \times 2,02$ & $\begin{array}{l}1868 \\
1872 \\
1879\end{array}$ & 2 & 1238 \\
\hline \multirow[t]{2}{*}{310} & $184 \times 098$ & $18 \times 24$ & MP-1239 & 45.I.1.153 & Sta. Casilda & & & & & \\
\hline & & $18 \times 24$ & $M P-1240$ & 25.2 .108 & $\begin{array}{l}\text { Niño Jesús dormido sobre una } \\
\text { Cruz. }\end{array}$ & & & & & \\
\hline 97 & $136 \times 167$ & $18 \times 24$ & $M P \mathbb{P}-1241$ & 25.2 .109 & $\begin{array}{l}\text { Hércules separando los dos } \\
\text { montes Calpe y Abyla }\end{array}$ & & & & & \\
\hline 98 & $1,36 \times 1,67$ & $18 \times 24$ & $M P \cdot 1242$ & 25.2 .110 & Hércules venciendo a Gerion & & & & & \\
\hline 99 & $131 \times 166$ & $18 \times 24$ & $M P \cdot 1243$ & 25.2 .111 & $\begin{array}{l}\text { Hércules luchando con el león } \\
\text { de la Selva Negra }\end{array}$ & & & & & \\
\hline 100 & $132 \times 153$ & $18 \times 24$ & $\mathrm{MP}-1244$ & 25.2 .112 & $\begin{array}{l}\text { Héroules luchando oon el } \\
\text { jabali de Erimanto }\end{array}$ & & & & & \\
\hline 101 & $133 \times 152$ & $18 \times 24$ & MP-1245 & 25.2 .113 & $\begin{array}{l}\text { Hércules sujetando al toro de } \\
\text { Creta que envió Neptuno } \\
\text { contra Minos }\end{array}$ & & & & & \\
\hline 102 & $136 x 151$ & $18 \times 24$ & MP-1246 & 25.2 .114 & Hércules luchando con Anteo & & & & & \\
\hline 103 & $132 \times 151$ & $18 \times 24$ & MP-1247 & 25.2 .115 & $\begin{array}{l}\text { Hércules luchando con el } \\
\text { cancerbero para sacar a } \\
\text { Alcestes del Infierno }\end{array}$ & & & & & \\
\hline 104 & $133 \times 153$ & $18 \times 24$ & MP-1248 & 25.2 .116 & $\begin{array}{l}\text { Hércules deteniendo el cauce } \\
\text { del ro Alfeo }\end{array}$ & & & & & \\
\hline 105 & $136 \times 167$ & $18 \times 24$ & MP-1249 & 25.2 .117 & $\begin{array}{l}\text { Hércules matando a la hidra de } \\
\text { los pantanos de Verus. }\end{array}$ & & & & & \\
\hline 106 & $136 \times 167$ & $18 \times 24$ & MP-1250 & 25.2 .118 & $\begin{array}{l}\text { Hércules atormentado por el } \\
\text { fuego de la túnioa del centauro } \\
\text { Neso }\end{array}$ & & & & & \\
\hline
\end{tabular}




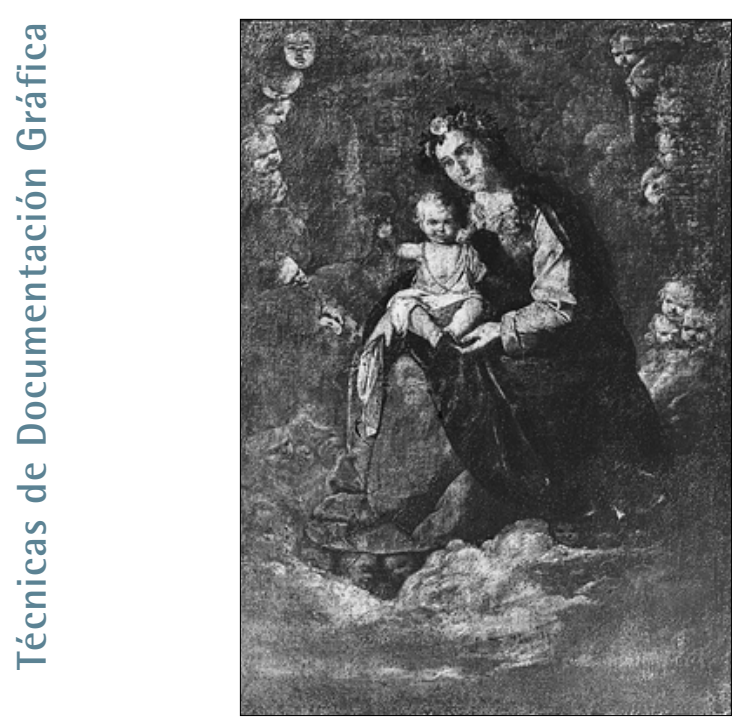

I. La Virgen del Rosario (N.I.M: 3289 A-1880)

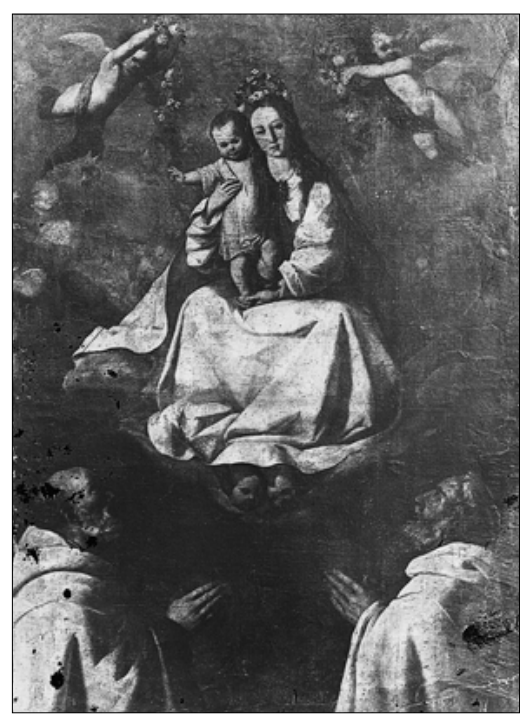

2. La Virgen de la Merced con diversos santos (N.I.M: 8751 A-1004)

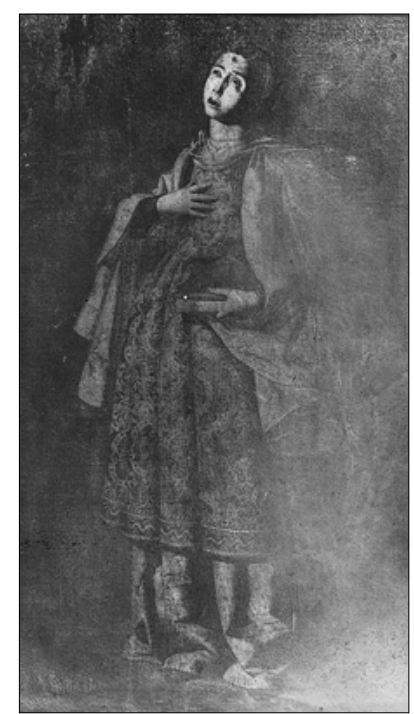

3. Santa Bárbara.

(N.I.M: $7808 \quad$ A-1059)

\section{ACADEMIA DE SAN FERNANDO}

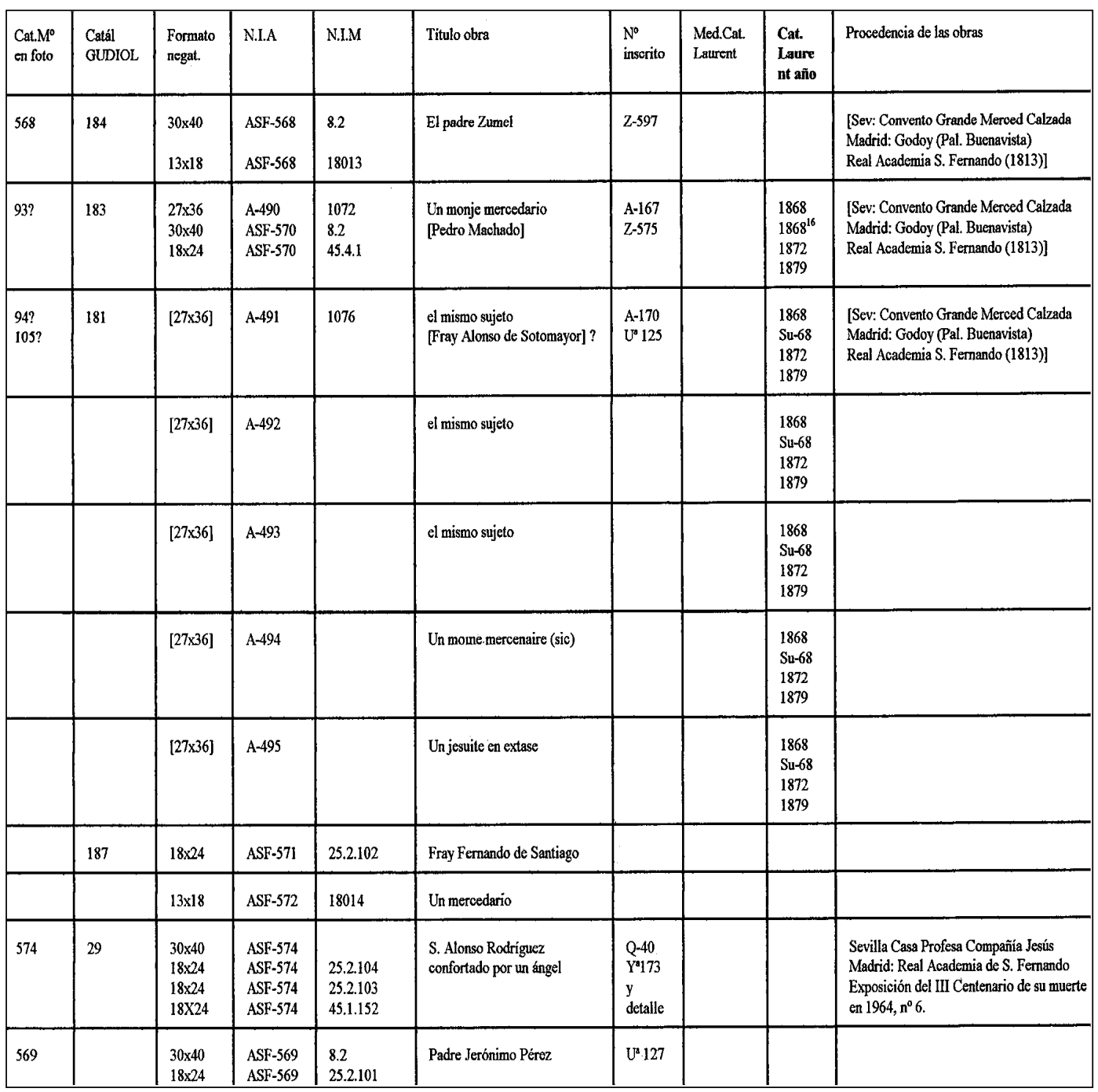




\section{OTROS. CUADROS EN EL MUSEO DE CÁDIZ QUE TAMBIÉN FOTOGRAFÍ́}

\begin{tabular}{|c|c|c|c|c|c|}
\hline \begin{tabular}{|l} 
Cat $\mathrm{M}^{\circ}$ \\
1835
\end{tabular} & $\begin{array}{l}\text { Formato } \\
\text { negativo }\end{array}$ & N.I.A & N.L.M & Titulo obra & Año catál Laurent \\
\hline & $27 \times 36$ & A-1816 & 3377 & [Murillo- Ecce Homo] & 1879 \\
\hline 27 & $\begin{array}{l}27 \times 36 \\
27 \times 36\end{array}$ & $\mathrm{~A}-1817$ & $\begin{array}{l}3380 \\
2232\end{array}$ & L. GIORDANO-Saint Michelè & 1879 \\
\hline 28 & $\begin{array}{l}27 \times 36 \\
27 \times 36\end{array}$ & A-1818 & $\begin{array}{l}3536 \\
7849\end{array}$ & L. GIORDANO-L'ange gardien & 1879 \\
\hline & $\begin{array}{l}27 \times 36 \\
27 \times 36\end{array}$ & A-1819 & $\begin{array}{l}3853 \\
2227\end{array}$ & [Signé N.P.-Le Jugement dernier] & 1879 \\
\hline$s / n$ & $\begin{array}{l}27 \times 36 \\
27 \times 36\end{array}$ & A-1831 & $\begin{array}{l}3852 \\
2236\end{array}$ & $\begin{array}{l}\text { Fernando Gallego-Le portement de croix.- Le Christ } \\
\text { mort-La Résurrection.- }\end{array}$ & 1879 \\
\hline 51 & $27 \times 36$ & A-1832 & 3379 & J.B. TIEPOLO-Femme avec un tambour de basque & 1879 \\
\hline 173 & $27 \times 36$ & $\mathrm{~A}-1833$ & 3851 & F. GOYA-Un majo & 1879 \\
\hline $\begin{array}{l}8 \\
\text { papel }\end{array}$ & $27 \times 36$ & A-1834 & 3580 & F. GOYA-Une maja & 1879 \\
\hline$s / n$ & $27 \times 36$ & A-1 1835 & 3581 & $\begin{array}{l}\text { ECOLE DE COLOGNE- La Vierge donnant le sein á } \\
\text { son divin Fils. }\end{array}$ & 1879 \\
\hline$s / n$ & $27 \times 36$ & A-1 1836 & 3583 & $\begin{array}{l}\text { A Ferrant y Fischermans- Le peintre Murillo tombant } \\
\text { d'un échafandage á Cádiz }\end{array}$ & 1879 \\
\hline $\sin$ & $\begin{array}{l}27 \times 36 \\
27 \times 36\end{array}$ & A-1 837 & $\begin{array}{l}116 \\
2237\end{array}$ & M. CABRAL Y BEJARANO- La caída de Murillo & 1879 \\
\hline $\sin$ & $27 \times 36$ & A-1 838 & 3382 & $\begin{array}{l}\text { M. Garcia Barcia-Consécration de la cathédrale de } \\
\text { Cádiz. }\end{array}$ & 1879 \\
\hline $3 / \mathbf{n}$ & $27 \times 36$ & $\mathrm{C}-2061$ & 8860 & Vista de la sala de esculturas del Museo & 1879 \\
\hline
\end{tabular}


CUADROS DE ZURBRÁN FOTOGRAFIADOS POR LAURENT Y OTROS FOTÓGRAFOS DEL ARCHIVO:

\begin{tabular}{|c|c|c|c|c|c|c|c|c|c|}
\hline $\begin{array}{l}\text { Cat.M } \\
0 . \\
1835\end{array}$ & $\begin{array}{l}\text { Cat. } \\
\text { Gutdiol }\end{array}$ & $\begin{array}{l}\text { Formato } \\
\text { soporte }\end{array}$ & N.IA & N.IM & Título obra & Reserv & Flecha & $\begin{array}{l}\text { MEDDA } \\
\text { Pemán }\end{array}$ & $\begin{array}{l}\text { Año Catälogo } \\
\text { Laurent } \\
\text { Procedencia de las } \\
\text { obras }\end{array}$ \\
\hline [63] & 213 & $\begin{array}{l}{[27 \times 36]} \\
30 \times 40\end{array}$ & $\begin{array}{l}\text { A-1 } 1822 \\
55838\end{array}$ & & La Porciúncula & Q39 & & $\begin{array}{l}2485 \times 1672 \\
\text { Cat. } 1964 . \\
\text { Fot RV }\end{array}$ & $\begin{array}{l}\text { Conv. Capuchinos } \\
\text { Jerez Frontera } \\
\text { M Prov. Cádiz }^{\circ}\end{array}$ \\
\hline [64] & 127 & $\begin{array}{l}27 \times 36 \\
27 \times 36\end{array}$ & A-1829 & $\begin{array}{l}2228 \\
3863\end{array}$ & S. Brumo en oración & & $\begin{array}{l}1 / 2 \\
\text { punto }\end{array}$ & $\begin{array}{l}3414 \times 1950 \\
\text { Cat.1964. } \\
\text { Fot RV }\end{array}$ & 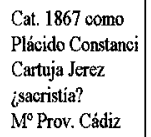 \\
\hline & & $\begin{array}{l}27 \times 36 \\
27 \times 36\end{array}$ & $\begin{array}{l}\text { A-1806 } \\
\text { A-1806 }\end{array}$ & $\begin{array}{l}9078 \\
9151\end{array}$ & Santa Catalina & & & & $\begin{array}{l}\text { Catedral de } \\
\text { Palencia }\end{array}$ \\
\hline & 319 & $27 \times 36$ & A-1880 & 3289 & La Virgen del Rosario & & & & $\begin{array}{l}\text { Catedral de } \\
\text { Sevilla }\end{array}$ \\
\hline
\end{tabular}



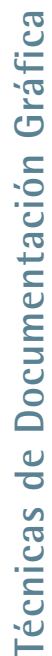

4. Santo Tomás de Aquino. (N.IM: 688 A-1090)
5. El Milagro de San Hugo en el refrectorio. (N.I.M: 907 A-1086)

6. San Bruno hablando con el Papa. (N.I.M: 8488 A-1098) 


\section{OBRAS DE ZURBARÁN O ATRIBUIDAS A ÉL, POR OTROS FOTÓGRAFOS DEL ARCHIVO}

\begin{tabular}{|c|c|c|c|c|c|c|c|}
\hline $\begin{array}{l}\text { GUDIO } \\
\mathrm{L}\end{array}$ & $\begin{array}{l}\text { Medida } \\
\text { Gudiol }\end{array}$ & N.I.A & N.I.M & Formato placa & TITULO & $\begin{array}{l}\text { Medida } \\
\text { FORV }^{17}\end{array}$ & Procedencia del cuadro \\
\hline \multirow[t]{10}{*}{256} & $110 \times 084$ & 11181 & & $30 \times 40$ & San Diego de Alcalá & $117 \times 087$ & $\begin{array}{l}\text { Col. Lázaro } \\
\text { [Madrid } \text { M }^{\circ} \text { Lázaro] }\end{array}$ \\
\hline & & 11515 & & $18 \times 24$ & $\begin{array}{l}\text { Santa Catalina depositada por los } \\
\text { ángeles }\end{array}$ & & Col. Lázaro. Madrid. \\
\hline & & 12178 & 45.2.179 & $\begin{array}{l}18 \times 24 \\
C_{j} 74 r 8\end{array}$ & Sagrada Familia & & Col. Lázaro \\
\hline & & 12179 & $\begin{array}{l}15506 \\
45.2 .180\end{array}$ & $\begin{array}{l}18 \times 24 \\
\mathrm{Cj} 58 \mathrm{r} 13\end{array}$ & La Inmaculada Concepción & & Col. Lázaro. Madrid. \\
\hline & & 12432 & 38.1 .143 & $\begin{array}{l}18 \times 24 \\
\mathrm{Cj} 69 \mathrm{r} 12\end{array}$ & Un santo & & Col. Lázaro. Madrid. \\
\hline & & 13386 & & $9 \times 12$ & Virgen y niño & & $\begin{array}{l}\text { Col. Georges Loukine, C/Rafael Calvo, 22-Madrid, } \\
\text { Hay certificado del cuadro por D. Fernando } \\
\text { Labrada, con el n } \text { n }^{\circ} 31691 \\
\text { El 22-1-1942, pasa a depender de D. Tomás Aldey } \\
\text { Redonet. Fotografiado 16-10-1941. }\end{array}$ \\
\hline & & 13387 & & $9 \times 12$ & $\begin{array}{l}\text { detalle del } 13886 \text { [Virgen con el } \\
\text { Nif̂̃o] }\end{array}$ & & Fotografiado 16-10-1941. \\
\hline & & 13388 & & $9 \times 12$ & $\begin{array}{l}\text { Firma de Zurbarain, en el cuadro } \\
13386 \text { [Virgen con el Nifito] }\end{array}$ & & $\begin{array}{l}\text { Col. Georges Loukine, C/Rafael Calvo, 22-Madrid, } \\
\text { El 22-1-1942, pasa a depender de D. Tomás Aldey } \\
\text { Redonet. Fotografiado 16-10-1941. }\end{array}$ \\
\hline & & 31691 & & $18 \times 24$ & $\begin{array}{l}\text { certificado del cuadro por D. } \\
\text { Fernando Labrada, con el no } \mathrm{n}^{\circ} 31691 \\
\text { [Virgen con el Niño] }\end{array}$ & & \\
\hline & & 30071 & 25.2 .150 & $\begin{array}{l}18 \times 24 \\
C j 8 r 31\end{array}$ & S. Francisco & $075 \times 058$ & Prop. Eduardo Martinez del Campo \\
\hline \multirow[t]{2}{*}{522} & $167 \times 107$ & $\begin{array}{l}30074 \\
53648\end{array}$ & 25.2 .141 & $\begin{array}{l}18 \times 24 \\
30 \times 40\end{array}$ & $\begin{array}{l}\text { Cristo en la columna, última obra } \\
\text { conocida }\end{array}$ & $167 \times 107$ & $\begin{array}{l}\text { Parroquia de San Juan Bautista. Jadraque } \\
\text { [catalogo Expo Zurbarán en el III centenario de su } \\
\text { muerte, Madrid, 1964, } \mathrm{n}^{\circ} \text { 103] }\end{array}$ \\
\hline & & 30089 & 25.2 .157 & $\begin{array}{l}18 \times 24 \\
\mathrm{Cj} 8 \mathrm{r} 17\end{array}$ & La Santa Faz & $101 \times 078$ & Prop. D. Mariano Pacheco \\
\hline \multirow[t]{3}{*}{527} & $291 \times 165$ & 30091 & & $\begin{array}{l}18 \times 24 \\
\text { Cj del MoP } \\
2472\end{array}$ & San Jacobo de la Marca & & $\begin{array}{l}\text { [Alcalá de Henares: Convento de S. Diego } \\
\text { Madrid: Mo de la Trinidad] } \\
\text { Igl. S. Francisco el Grande } \\
\text { [Iglesia S. Fco el Grande } \\
M^{\circ} \text { del Prado } n^{\circ} 2472 \text { ] }\end{array}$ \\
\hline & & 30092 & 25.2 .167 & $\begin{array}{l}18 \times 24 \\
\mathrm{Cj} 7 \mathrm{r6}\end{array}$ & San Buenaventura & $290 \times 165$ & Iglesia de S. Francisco el Grande. Madrid. \\
\hline & & 30095 & 25.2 .153 & $\begin{array}{l}18 \times 24 \\
\mathrm{Cj} 8 \times 22\end{array}$ & S. Francisco de Paula & & Prop. D. J. Mac Dongall. Sevilla \\
\hline 551 & $203 \times 135$ & 30096 & 25.2 .146 & $\begin{array}{l}18 \times 24 \\
\mathrm{Cj} 8 \mathrm{r} 4 \mathrm{I}\end{array}$ & Santo Domingo de Guzmán & $203 \times 135$ & $\begin{array}{l}\text { [Sevilla: Francisco Romero Comavachuelo] } \\
\text { Prop. D. Franciseo de Romero Canavechuelo y } \\
\text { Balmaseda (Sevilla) } \\
\text { [Francisco Romero Brunet } \\
\text { Bilbao: Félix Valdés } \\
\text { Colección Zubillaga] }\end{array}$ \\
\hline \multirow[t]{3}{*}{$58 ?$} & $123 \times 083$ & 30097 & 25.2 .154 & $\begin{array}{l}18 \times 24 \\
\mathrm{Cj}_{\mathrm{j}} 8 \mathrm{r} 21\end{array}$ & $\begin{array}{l}\text { El Niño Jesús herido al tejer una } \\
\text { corona de Espinas }\end{array}$ & $102 \times 073$ & $\begin{array}{l}\text { [Jerez: col partic.; Madrid: Joaquin Payá] } \\
\text { Prop. Vizconde del Castillo del Genovés. }\end{array}$ \\
\hline & & $\begin{array}{l}30098 \\
\text { MP2804 }\end{array}$ & & $\begin{array}{l}18 \times 24 \\
\text { Cj } 12 \text { r6 }\end{array}$ & Jesus $\mathrm{y}$ un santo & & Prop. D. Rafael Martinez \\
\hline & & MP2803 & & $13 \times 18$ & Bodegón & & Donativo F. Cambo, 1940 al M del Prado. Madrid. \\
\hline \multirow[t]{3}{*}{186} & $198 \times 124$ & 30104 & 25.2 .151 & $\begin{array}{l}18 \times 24 \\
\mathrm{Cj} 8+25\end{array}$ & $\begin{array}{l}\text { San Carmelo [obispo de Teruel] } \\
\text { [San Leandro] }\end{array}$ & & $\begin{array}{l}\text { [Sevilla: Convento Grande de la Merced (Libreria)] } \\
\text { Parroquia de Sta. Bárbara }\end{array}$ \\
\hline & & 30107 & 25.2 .145 & $18 \times 24$ & S. Francisco Javier & & Iglesia de S. Francisco el Grande. Madrid. \\
\hline & & 30931 & & & Cristo & & Colección particular, desconocida. \\
\hline 259 & $105 \times 084$ & 30110 & 45.4 .4 & $\begin{array}{l}18 \times 24^{18} \\
18 \times 24\end{array}$ & San Lucas ante Cristo Crucificado & & $\begin{array}{l}\text { Madrid: posiblemente en las Colecciones Reales. } \\
\text { Pau: Infante Sebastiän Gabriel (+1875, bisnicto de }\end{array}$ \\
\hline
\end{tabular}




\section{OBRAS DE ZURBARÁN O ATRIBUIDAS A ÉL, POR OTROS FOTÓGRAFOS DEL ARCHIVO}

\begin{tabular}{|c|c|c|c|c|c|c|c|}
\hline $\begin{array}{l}\text { GUDIO } \\
\text { L }\end{array}$ & $\begin{array}{l}\text { Medida } \\
\text { Gudiol }\end{array}$ & N.I.A & N.I.M & Formato placa & TTTULO & $\begin{array}{l}\text { Medida } \\
\text { FORV }^{17}\end{array}$ & Procedencia del cuadro \\
\hline & & & & & & & $\begin{array}{l}\text { Carlos III) } \\
\text { Madrid: Cristóbal Colón, Duque de Veragua (casado } \\
\text { con la heredera del Infante Sebastián Gabriel) } \\
M^{0} \text { del Prado no } 2594 \text { (1936)] } \\
\text { este mismo asunto existe actualmente en el } M^{0} \text { Prado } \\
\text { con el no } 2594 \text {. }\end{array}$ \\
\hline 214 & $117 \times 094$ & 30132 & 25.2 .171 & $18 \times 24$ & La Virgen Niña & & $\begin{array}{l}\text { [Madrid] propiedad de D. Aureliano Beruete] } \\
\text { [Dario de Regoyos] } \\
\text { [New York: Metropolitan Museum of Art no } 27- \\
\text { 137] }\end{array}$ \\
\hline 250 & $170 \times 108$ & 32987 & 14316 & $12 \times 16$ & Autorretrato & & $\mathrm{M}^{0}$ de Braunwich \\
\hline \multirow[t]{3}{*}{28} & $174 \times 138$ & 32898 & & & Inmaculada & $174 \times 138$ & $\begin{array}{l}\text { [SEVILLA: Cabildo Municipal. } 1630 \\
\text { Madrid: Melchor Gaspar de Jovellanos:1744-1811; } \\
\text { Juan Arias Saavedra (Testamentaria de Jovellanos)] } \\
\text { Legado por M Cristina Pelado y Verdugo, biznieta } \\
\text { de Arias Saavedra en } 1952 \text { al ] Colegio Parroquial } \\
\text { de Ntra. Sra. del Carmen de Jadraquc. }\end{array}$ \\
\hline & & 49075 & 15297 & $9 \times 12$ & Flores & & Colección Sr. Martinez de la Vega \\
\hline & & 49143 & & & [Busto de un religioso] & & $\begin{array}{l}\text { Prod. Sra. de Villalón } \\
\text { Fomento, } 11 \\
\text { Fot Abril 1955, por JA }\end{array}$ \\
\hline 149 & $290 \times 222$ & 49176 & & & Aparición de Jesús al Padre Salmerón & & [Sacristia del Monasterio de Guadalupe] \\
\hline 147 & $290 \times 222$ & 49200 & & $6 \times 6$ & Misa del Padre Cabañuelas & & [Sacristia del Monasterio de Guadalupe] \\
\hline \multirow[t]{2}{*}{151} & & $49201 \mathrm{bis}$ & & $6 \times 6$ & $\begin{array}{l}\text { Fray Martin Vizoaino repartiendo } \\
\text { limosnas }\end{array}$ & & Monasterio de Guadalupe \\
\hline & & 49588 & 11489 & $9 \times 12$ & $\begin{array}{l}\text { San Serapio (reproducción de una } \\
\text { lámina) Fot Abril 1953, por J A }\end{array}$ & & Hilford Wadswork Atheneum \\
\hline \multirow[t]{2}{*}{268} & & 49589 & 11487 & $9 \times 12$ & $\begin{array}{l}\text { Sta. Catalina dépositada por los } \\
\text { ángeles [es El Tránsito de la Virgen] }\end{array}$ & & Reproduce de una lámina en abril de 1953 por JA \\
\hline & & 49639 & & $13 \times 18^{19}$ & Adoración & & $\begin{array}{l}\text { Prod. Sr. Muguiro } \\
\text { Velázquez, } 67 \\
\text { fot Abril } 1953\end{array}$ \\
\hline \multirow[t]{3}{*}{243} & & 50095 & & $\begin{array}{l}13 \times 18 \text { por } \\
\text { J.A. }\end{array}$ & $\begin{array}{l}\text { Cabeza de S. José [fragmento de la A- } \\
\text { 1087] }\end{array}$ & & Fot. enero 1952 por J.A. \\
\hline & & 50929 & 27.1.119 & $18 \times 24$ & Los discipulos de Emaús & & $\begin{array}{l}\text { Propiedad del Dr. Vega Diaz. Fot 28-3-195I, en } \\
\text { Serrano } 62 \text {. No hay negativo }\end{array}$ \\
\hline & & 51716 & 14992 & $9 \times 12$ & El escultor ciego & & Propiedad de D. Luis Pérez Bueno \\
\hline \multirow[t]{7}{*}{$\$ 21 \%$} & & 51874 & 15023 & $\begin{array}{l}13 \times 18 \\
\text { (reprod) }\end{array}$ & $\begin{array}{l}\text { San Pedro Nolasco en una } \\
\text { oirounstanoia de su vida }\end{array}$ & & Fot en Sept. 1948, en el taller, de una reproducción \\
\hline & & 52100 & $\begin{array}{l}27.2 .173 \\
27.2 .174\end{array}$ & $\begin{array}{l}18 \times 24 \text { por JA } \\
18 \times 24\end{array}$ & S. Francisco & & $\begin{array}{l}\text { Prod. de la Sra. de Bucharo } \\
\text { Rey Franciseo, } 3 \text {. } \\
\text { Fot Abril } 1948\end{array}$ \\
\hline & & 54080 & 14316 & $12 \times 16$ & Autorretrato y firma de Zurbarán & & $\mathrm{M}^{0}$ de Braunwich \\
\hline & & 54606 & & $24 \times 30$ & Cristo atado a la columna & & $\begin{array}{l}\text { Col. D. José Ricardo Gómez Acebo } \\
\text { Fot. 28-1-1935 }\end{array}$ \\
\hline & & 54649 & 27.3.111 & $18 \times 24$ & Adoración de los Pastores & & $\mathrm{M}^{0}$ Provincial de Córdoba. \\
\hline & & 54738 & 14390 & & Santa Agueda & & Colección Sra. Vda de Domínguez. \\
\hline & & 54808 & 14346 & $9 \times 12$ & $\begin{array}{l}\text { Retrato de una niña (copia de } \\
\text { Zurbarán) }\end{array}$ & & Col. Sr. Sallent o Sayent \\
\hline
\end{tabular}




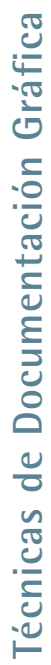



7. [Retrato de] Un mercedario [Madrid] (T.M: 25.2. 147 30073)

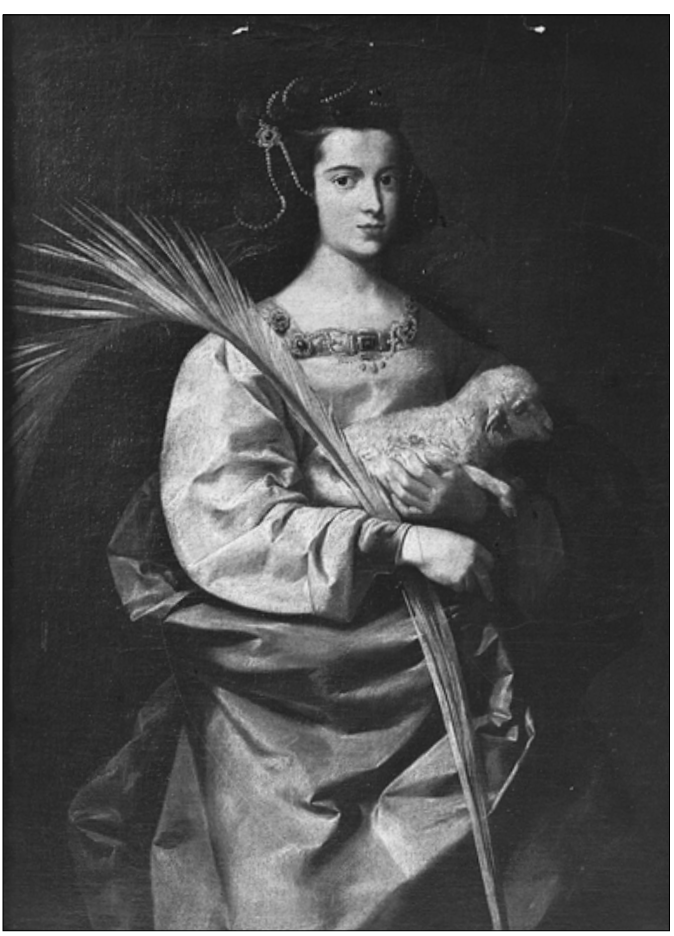

8. Santa Inés

(T.M: 25.2.160 30087)

OBRAS DE ZURBARÁN O ATRIBUIDAS A ÉL, POR OTROS FOTÓGRAFOS DEL ARCHIVO

\begin{tabular}{|c|c|c|c|c|c|c|}
\hline & & 65275 & $44,4.220$ & $\begin{array}{l}\text { Pelc Isopan } \\
13 \times 18 \\
\text { para } 18 \times 24\end{array}$ & $\begin{array}{l}\text { S. Jerónimo dando Is Comunión a Sta. } \\
\text { Marria Egippiaca }\end{array}$ & $\begin{array}{l}\text { Encargo del Sr. Serrano, de la CModesto Lafuente, } \\
\text { 50. Derechos reservados por el propietario. } \\
\text { Hecho en Febrero de } 1940\end{array}$ \\
\hline & & 65465 & & $\begin{array}{l}13 \times 18 \\
\text { para } 18 \times 24\end{array}$ & S. Francisco en éxtasis & $\begin{array}{l}\text { Prop. del Sr. Sopranis } \\
\text { C/ODonnell,3. Mayo } 1941\end{array}$ \\
\hline & & MS-190 & 25.2 .120 & $\begin{array}{l}18 \times 24 \\
\text { Cj28 del } \\
\text { M० de Sev }^{\circ}\end{array}$ & $\begin{array}{l}\text { S. Francisco de Borja, duque de } \\
\text { Gandía }\end{array}$ & $M^{\circ}$ de Sevilla \\
\hline & & MS-189 & 25.2 .119 & $18 \times 24$ & $\begin{array}{l}\text { S. Francisco de Boriag } \\
\text { Actualumente atribuido a Alonso } \\
\text { CANO }\end{array}$ & $M^{\circ}$ de Sevilla \\
\hline & & MS-192 & 25.2 .121 & $\begin{array}{l}18 \times 24 \\
\text { Caja Mo Sev }\end{array}$ & Santo Obispo & \\
\hline & & MS-193 & 25.2 .122 & $18 \times 24 \mathrm{M}^{\circ} \mathrm{Sev}$ & S. Jerónimo & \\
\hline & & MS-194 & 26.2 .77 & $18 \times 24$ & La virgen & \\
\hline & & MS-19S & 29.2 & $\begin{array}{l}18 \times 24 \\
M^{0} \mathrm{sev}\end{array}$ & Cristo de la expiración & \\
\hline & & MS-196 & 25.2.124 & $18 \times 24 \mathrm{M}^{\circ} \mathrm{Sev}$ & Santo Obispo & \\
\hline & & MS-199 & 26.2 .76 & $18 \times 24$ & Apoteosis de Sto Tomás de Aquino & \\
\hline
\end{tabular}




\section{OBRAS DE ZURBARÁN O ATRIBUIDAS A ÉL, POR OTROS FOTÓGRAFOS DEL ARCHIVO}

\begin{tabular}{|c|c|c|c|c|c|c|c|c|}
\hline \begin{tabular}{|l}
$\mathrm{N}^{0}$ Cat \\
$\mathrm{M}^{\mathrm{p}}$
\end{tabular} & $\begin{array}{l}\text { Catálogo } \\
\text { GUDIOL }\end{array}$ & $\begin{array}{l}\text { Medida } \\
\text { GUDIOL }\end{array}$ & NIA & NIM & $\begin{array}{l}\text { Formato } \\
\text { placa }\end{array}$ & TITULO & $\begin{array}{l}\text { Medida } \\
\text { FORV }\end{array}$ & Procedencia cuadro \\
\hline & & & 12249 & & & $\begin{array}{l}\text { La Virgen del Rosario } \\
\text { (Catálogo Lacoste de 1914) }\end{array}$ & $110 \times 138$ & Casa Torres \\
\hline & & & [12252] & & $30 \times 40$ & $\begin{array}{l}\text { Niño dormido } \\
\text { (Catálogo Lacoste de 1914) }\end{array}$ & $050 \times 075$ & Casa Torres \\
\hline & & & 12254 & & & $\begin{array}{l}\text { Retrato de un cardenal } \\
\text { (Catálogo Lacoste de 1914) }\end{array}$ & $137 \times 214$ & Casa Torres \\
\hline & & & 12255 & & $\begin{array}{l}18 \times 24 \\
\mathrm{Cj} 13, \mathrm{rl} 3\end{array}$ & $\begin{array}{l}\text { S. Ignacio de Loyola (estilo de } \\
\text { Zurbarán) } \\
\text { (Catálogo Lacoste de 1914) }\end{array}$ & $027 \times 036$ & Casa Torres \\
\hline & & & 30069 & 25.2 .139 & $18 \times 24$ & Sta. Catalina & & $\begin{array}{l}\text { Legado del Marqués de Altamira a S.M. en prueba } \\
\text { de respeto y lealtad. } \\
\text { Propiedad D. Alfonso de Borbón }\end{array}$ \\
\hline & & & 30070 & & $\begin{array}{l}18 \times 24 \\
\mathrm{Cj} 12, \mathrm{r} 26\end{array}$ & Las lágrimas de S. Pedro & & Propiedad D. Pedro Flores \\
\hline & 520 & $127 \times 097$ & 30072 & 31.3 .122 & $\begin{array}{l}18 \times 24 \\
\dot{C} \mathrm{j} 10, \mathrm{r} 77\end{array}$ & S. Francisco [arrodillado] & $127 \times 097$ & $\begin{array}{l}\text { Propiedad D. Aureliano Beruete[Madrid (1905)] } \\
\text { [Bilbao. Félix Valdés (h.1945)] }\end{array}$ \\
\hline & 178 & $069 \times 056$ & 30073 & 25.2 .147 & $18 \times 24$ & $\begin{array}{l}\text { [Retrato de] Un mercedario } \\
\text { [Madrid] }\end{array}$ & & $\begin{array}{l}\text { Prod. Marqués de [Casa] Argudin. [1905] } \\
\text { [Rumeu de Armas] }\end{array}$ \\
\hline & 473 & $148 \times 108$ & 30075 & 25.2 .163 & $18 \times 24$ & S: Antonio [de Padua] & $149 \times 107$ & $\begin{array}{l}\text { [Manzanilla (Sevilla).Col. particular (S.XVIII)] } \\
\text { [Sevilla] Propiedad Salvador Cumplido [1905] } \\
\text { [Alfonso Grosso } \\
\text { Madrid. Mo del Prado (1958)] }\end{array}$ \\
\hline & 493 & $200 \times 104$ & 30076 & & $\begin{array}{l}18 \times 24 \\
\mathrm{Cj} 7, \mathrm{r} 1\end{array}$ & D. Diego Bustos de Lara & $200 \times 104$ & $\begin{array}{l}\text { [Sevilla: ¿Juana)Arias de Saavedra } \\
\text { Marqués de Moscoso] } \\
\text { Prod. Conde Gomara, Sevilla theredó el cuadro del } \\
\text { anterior propietario] } \\
\text { [Conde Bustillo] }\end{array}$ \\
\hline & 498 & $200 \times 104$ & 30077 & 31.3 .81 & $\begin{array}{l}18 \times 24 \\
\mathrm{Cj} 10, \mathrm{R} 96\end{array}$ & D. Gonzalo Bustos de Lara & $200 \times 104$ & $\begin{array}{l}\text { [Sevilla: Joaquin Albarracin (hijo de Juana Arias } \\
\text { de Saavedra] } \\
\text { Prop. Conde de Gomara, Sevilla }\end{array}$ \\
\hline & 313 & $200 \times 145$ & 30078 & 31.3 .75 & $\begin{array}{l}18 \times 24 \\
\text { Cj } 8, \mathbf{r} 29\end{array}$ & La Concepción & $200 \times 146$ & $\begin{array}{l}\text { [Madrid Marqués de Leganés (S. XVII)] } \\
\text { [Paris: Venta Marqués de Salamanca no 43, (1875)] } \\
\text { Prop. Marqués de Cerralbo, Museo. }\end{array}$ \\
\hline & 359 & $190 \times 110$ & 30079 & 31.4 .21 & $\begin{array}{l}18 \times 24 \\
\mathrm{Cj} 1, \mathrm{r} 82\end{array}$ & S. Agustin & $190 \times 110$ & $\begin{array}{l}\text { Convento de los Capuchinos de Casteflión. [Legado } \\
\text { de la Condesa de Campo Alange]. }\end{array}$ \\
\hline & 356 & $190 \times 110$ & $\begin{array}{l}30080 \\
\text { MS-208 }\end{array}$ & $\begin{array}{l}25.2 .165 \\
25.2 .136\end{array}$ & $\begin{array}{l}18 \times 24 \\
\mathrm{Cj} 7, \mathrm{r} 4\end{array}$ & Santo Domingo & $190 \times 110$ & $\begin{array}{l}\text { Convento de los Capuchinos de Castellón. } \\
\text { [Legado de la Condesa de Campo Alange]. }\end{array}$ \\
\hline & 353 & $190 \times 110$ & 30081 & 25.2 .166 & $18 \times 24$ & S. Bruno & $190 \times 110$ & $\begin{array}{l}\text { Convento de los Capuchinos de Castellón. } \\
\text { [Legado de la Condesa de Campo Alange]. }\end{array}$ \\
\hline & 358 & $190 \times 110$ & 30082 & 25.2 .168 & $18 \times 24$ & S. Ignacio de Loyola & $190 \times 110$ & $\begin{array}{l}\text { Convento de las Capuchinas de Castellón } \\
\text { [Legado de la Condesa de Campo Alange]. }\end{array}$ \\
\hline & 354 & $190 \times 110$ & 30083 & 25.2 .169 & $18 \times 24$ & S. Francisco & $190 \times 110$ & $\begin{array}{l}\text { Convento de los Capuchinos de Castellón. } \\
\text { [Legado de la Condess de Campo Alange]. }\end{array}$ \\
\hline & 352 & $190 \times 110$ & 30084 & 25.2 .170 & $18 \times 24$ & S. Pedro Nolasco & 190x110 & $\begin{array}{l}\text { Convento de los Capuchinos de Castellón. } \\
\text { [Legado de la Condesa de Campo Alange]. }\end{array}$ \\
\hline & 357 & $190 \times 110$ & 30085 & 25.2 .164 & $18 \times 24$ & S. Basilio & $190 \times 110$ & $\begin{array}{l}\text { Convento de los Capuchinos de Castellón. } \\
\text { [Legado de la Condesa de Campo Alange]. }\end{array}$ \\
\hline & 361 & $190 \times 110$ & 30086 & 25.2.162 & $18 \times 24$ & San Benito & $190 \times 110$ & $\begin{array}{l}\text { Convento de los Capuchinos de Castellón. } \\
\text { [Legado de la Condesa de Campo Alange]. }\end{array}$ \\
\hline & 457 & $146 \times 108$ & 30087 & 25.2 .160 & $18 \times 24$ & Santa Inós & $146 \times 108$ & $\begin{array}{l}\text { [Madrid] Prop. Duque de Béjar } \\
\text { [Lugano: Barón Henry Thissen-Bornemisza] }\end{array}$ \\
\hline & 557 & $109 \times 077$ & 30088 & 25.2 .159 & $18 \times 24$ & Paño de la Verónica & $105 \times 077$ & Prod. D. Angel Avilés [Madrid] \\
\hline & 48 & $168 \times 119$ & 30090 & 25.2 .156 & $18 \times 24$ & Cristo crucificado & $168 \times 086$ & $\begin{array}{l}\text { [Madrid] Manuel de Longoria } \\
\text { [Condesa de Liniers } \\
\text { Colección Part. New York] }\end{array}$ \\
\hline & 198 & $061 \times 041$ & 30093 & 25.2 .140 & $18 \times 24$ & $\begin{array}{l}\text { Fraile cartujo [fraile } \\
\text { crucificado] }\end{array}$ & $062 \times 042$ & $\begin{array}{l}\text { [Sevilla: Convento de S. José de la Merced } \\
\text { Descalza } \\
\text { Inventario de los cuadros del Alcázar de Sevilla en } \\
\text { 1810. vor orden de José Bonavarte. abareen } 38\end{array}$ \\
\hline
\end{tabular}


OBRAS DE ZURBARÁN O ATRIBUIDAS A ÉL, POR OTROS FOTÓGRAFOS DEL ARCHIVO

\begin{tabular}{|c|c|c|c|c|c|c|c|c|}
\hline & & & 30094 & 25.2 .152 & $18 \times 24$ & Flores y frutas & $082 \times 109$ & Prop. Condesa de Montareo \\
\hline & & & 30099 & 25.2 .149 & $18 \times 24$ & S. Francisco & & sin datos \\
\hline & 326 & $185 \times 103$ & 30100 & 25.2 .144 & $18 \times 24$ & Un Santo [S. Jerónimo] & & $\begin{array}{l}\text { [Madrid. Barón Heytesbury }{ }^{\circ} 1357 \text { (1926) } \\
\text { Lucena: Bōhler \& Steinmeyer } \\
\text { New York: Kleinberger \& Co. } \\
\text { San Diego (California):Fine Arts Gallery (1940)] }\end{array}$ \\
\hline & 267 & $138 \times 104$ & 30105 & 25.2 .161 & $18 \times 24$ & $\begin{array}{l}\text { Dos ángeles mancebos } \\
\text { [Adoración de la Eucaristía] }\end{array}$ & $138 \times 104$ & $\begin{array}{l}\text { Prop. Isabel López } \\
\text { [Madrid: Herederos de Isabel López (1905) } \\
\text { Villanueva de Segura (Murcia, Hospicio. } \\
\text { Murcia: Museo Provincial de Bellas Artes] }\end{array}$ \\
\hline & 57 & $128 \times 085$ & 30106 & 25.2 .155 & $18 \times 24$ & $\begin{array}{l}\text { El Niño Jesús hiriéndose al } \\
\text { tejer una corona de espinas }\end{array}$ & $131 \times 086$ & $\begin{array}{l}\text { [Sevilla. según la tradición en la Cartuja de las } \\
\text { Cuevas] } \\
\text { Prop. D. Cayetano Sánchez Pineda } \\
\text { [Sevilla: Sanchez Ramos] }\end{array}$ \\
\hline & 191 & $062 \times 041$ & 30108 & 31.3 .83 & $\begin{array}{l}18 \times 24 \\
\mathrm{Cj} 10, \mathrm{r} 38\end{array}$ & Un monje cartujo & & $\begin{array}{l}\text { [Sevilla: Convento de S. José de la Merced } \\
\text { Descalza } \\
\text { Inventario de los cuadros del Alcázar de Sevilla en } \\
\text { 1810, por orden de José Bonaparte, aparecen } 38 \\
\text { "santos mecenarios (sic) mártires" de 3/4x1/2 varas, } \\
\text { bajo los n } \mathrm{n}^{\circ} 179,337,362 \text { y } 380 \text { ] } \\
\text { Prop. Marqués de la Vega Inclán } \\
\text { [Toledo: Casa del Greco] [Fundación de la Vega } \\
\text { Inclán, Inventario 1963: }{ }^{\circ} 55 \text { ] } \\
\text { [restaurado recientemente en el Instituto del } \\
\text { Patrimonio Histórico. } \\
\text { Español] }\end{array}$ \\
\hline & 355 & $190 \times 110$ & 30109 & 25.2 .148 & $18 \times 24$ & San Jerónimo & & $\begin{array}{l}\text { Convento de los Capuchinos de Castellón } \\
\text { [Legado de la Condesa de Campo Alange] }\end{array}$ \\
\hline & 1 & $193 \times 156$ & 30111 & 25.2 .158 & $\begin{array}{l}18 \times 24 \\
30 \times 40 / Y- \\
134\end{array}$ & La Concepción & & $\begin{array}{l}\text { [Sevilla: Venta Manuel López Cepero n }{ }^{\circ} 66 \\
\text { (1860)] } \\
\text { Col. Dña Dolores Muni, Vda de López Cerezo, } \\
\text { Sevilla } \\
\text { [Madrid: Fernando Labrada } \\
\text { Bilbao: Felix Valdés] }\end{array}$ \\
\hline & 121 & $100 \times 073$ & 30133 & 25.2 .143 & $\begin{array}{l}18 \times 24 \mathrm{Cj} \\
8 \mathrm{r} 76\end{array}$ & El Salvador & & $\begin{array}{l}\text { [Salvador Mundi] Prop. Srta Iturbe } \\
\text { [Madrid: Vda. de Parcent. } \\
\text { Bareelona: Félix Millet (1939)] }\end{array}$ \\
\hline & & & 30134 & 25.2 .138 & $18 \times 24$ & S. Francisco & & Prod. Dña. Leonor Alonso \\
\hline & & & 30499 & 31.4 .27 & $18 \times 24$ & La Virgen y El Niño & & \\
\hline & 157 & $145 \times 103$ & 31516 & 33.1 .144 & & Apoteosis de S. Jerónimo & & $\begin{array}{l}\text { Monasterio de Guadalupe } \\
\text { Exp. temporal Mº Prado } 1959\end{array}$ \\
\hline & 243 & $248 \times 166$ & 32758 & & & Cristo coronando a S. José & & $\begin{array}{l}\text { [Sevilla: Iglesia del Conv. S. Jośc, Merced } \\
\text { Całzada } \\
\text { Depósito en el Alcázar no } 73(1810)] \\
M^{\circ} \text { Bellas Artes de Sevilla [n } n^{\circ} 207 \text {, antes de 1840] }\end{array}$ \\
\hline & 543 & $044 \times 034$ & $\begin{array}{l}53161 \\
\text { MP-2888 }\end{array}$ & 27.2.29 & $\begin{array}{l}18 \times 24(2) \\
\text { Fot enero } \\
1946\end{array}$ & Florero & & $\begin{array}{l}\text { [Mälaga: Antonio Pons } \\
M^{0} \text { Prov. de Bellas Artes de Málaga, en depósito } \\
\text { Madrid: } \text { M }^{\circ} \text { del Prado no } 2888 \text { (1945)] } \\
\text { Exp. de Floreros y Bodegones en la pintura } \\
\text { española "Madrid, 1936, p.151 }\end{array}$ \\
\hline$[67]$ & 133 & $061 \times 081$ & 54637 & 27.3 .123 & $18 \times 24$ & S. Lorenzo & & $\begin{array}{l}\text { [Altar Mayor de la Cartuja de N. Sra. Sta. Ma de la } \\
\text { Defensión, Extramuros de Jerez de la Frontera] } \\
M^{\circ} \text { de Cádiz [n'67, 1835] }\end{array}$ \\
\hline [78] & 131 & $055 \times 053$ & 55833 & 27.5 .16 & $18 \times 24$ & S. Marcos [es S. Lucas] & & 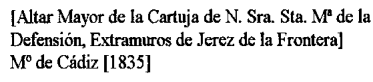 \\
\hline$[77]$ & 130 & $065 \times 063$ & 55834 & 27.5.17 & $18 \times 24$ & S. Juan Evangelista & & $\begin{array}{l}\text { [Altar Mayor de la Cartuja de N. Sra, Sta. Ma de la } \\
\text { Defensión, Extramuros de Jerez de la Frontera] } \\
\text { M de Cádiz [1835] }^{0}\end{array}$ \\
\hline [78] & 128 & $065 \times 063$ & 55835 & 27.5 .18 & $18 \times 24$ & San Mateo & &  \\
\hline [65] & 146 & $160 \times 116$ & 55836 & 25.2 .142 & $18 \times 24$ & Pentecostés & & $\mathrm{M}^{\circ}$ Provincial de Cádiz [1835] \\
\hline 79 & 129 & & 55837 & & $\begin{array}{l}\text { no hay } \\
\text { placa }\end{array}$ & $\begin{array}{l}\text { S. Mateo. [es S. Marcos, con } \\
\text { ángel] }\end{array}$ & & $\begin{array}{l}\text { Altar Mayor de la Carruja de Ntra. Sra. Sta. Me de } \\
\text { la Defensión, Extramuros de Jerez de la Fronteral }\end{array}$ \\
\hline \multirow[t]{2}{*}{66} & 132 & $061 \times 081$ & 55840 & & $18 \times 24$ & S. Juan Bautista & & $\begin{array}{l}\text { [Altar Mayor de la Cartuja de N. Sra. Sta. Ma de la } \\
\text { Defensión. Extramuros de Jerez de la Frontera } \\
\text { [Mo Romántico] }\end{array}$ \\
\hline & & & MP-1238 & 25.2 .7 & $\begin{array}{l}18 \times 24 \\
\mathrm{Cj} 13 \mathrm{MP} \\
27 \times 36 \\
\text { Uas43 }\end{array}$ & S. Francisco difunto & 080x190 & Mo Prado \\
\hline
\end{tabular}



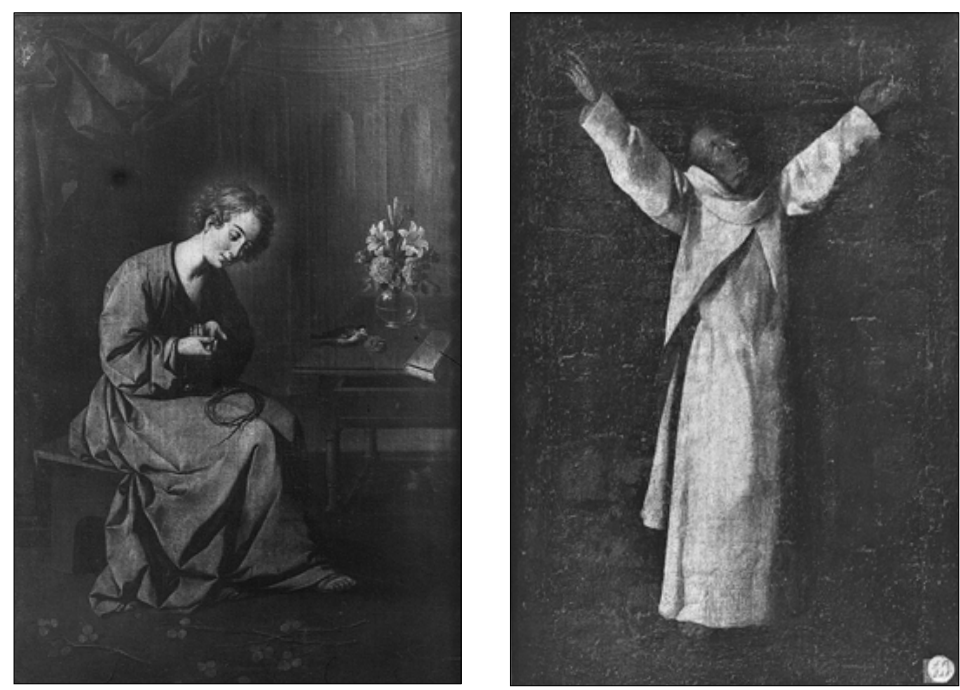

9. El Niño Jesús hiriéndose al tejer una corona de espinas.

(T.M: 25.2.155 30106)

10. Fraile cartujo [Fraile crucificado]. (T.M: 25.2.140 30093)

I I. Santa Catalina (T.M: 25.2.13930069)
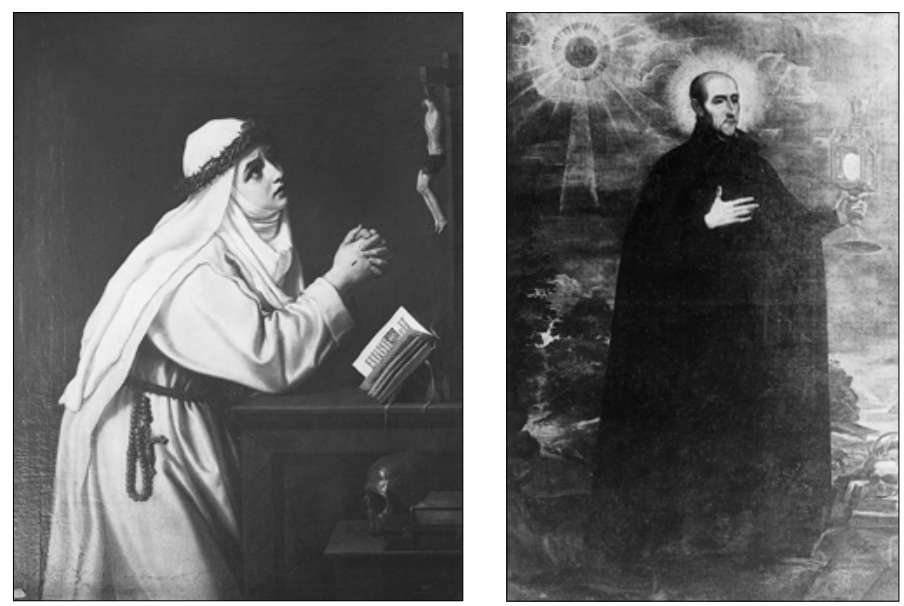

(T.M: 25.2.120 MS-190)

\section{Notas}

I. Se encuentra en Madrid, C/del Greco, 4.

2. Mal atribuido, ya que no es obra de Zurbarán.

3. La Virgen de la Merced con diversos Santos, La Anunciación, La Adoración de los pastores, La Adoración de los Reyes Magos, La Circuncisión y Un monje.

4. La Virgen y el Niño Jesús, Santa Eulalia, Santa Catalina, Santa Engracia, Santa Bárbara, Santa Matilde, Santa Inés, Santa Marina, Santa Dorotea.

5. Gutiérrez Martínez, Ana Ma - Laurent, de Jaspeador a Fotógrafo, en Un fotógrafo francés en la España del S. XIX. Mํ Educ y Cultura, $M^{\circ}$ Asuntos Exteriores, Caja Madrid y el Instituto Cervantes, Madrid, 1996, pp. 9-21.

6. Son autores de los negativos de los formatos de $27 \times 36 \mathrm{~cm}$, que fotografiaron en 1879.

7. No figura el autor en ellas, pero debió ser posiblemente Lacoste por el tamaño de los formatos y emulsiones utilizadas.

8. Nouveau Guide du touriste en Espagne et Portugal, J. Laurente et Cie Madrid, 1879.

9. Pemán en su Catálogo de 1964, reproduce una imagen como de Ruiz Vernacci, cuyo negativo se encuentra en los fondos de este Archivo.

10. Los N.I.A: A- 1004, A- 1005, A- 1006, A- 1007 y A- 1008 se mencionan en el Catálogo de la Colección del Duque de Montpensier, en 1866.
I I. [los N.I.A: A- I005, A- 1006 y A- 1008] Aparecían en el Catálogo de Laurent, pero no existe negativo en el Archivo.

12. Fue el antiguo Convento de la Merced, quemado por los franceses en 1810. Se formó como Museo tras la supresión de los conventos y fundaciones religiosas. Desde 1872 la Academia de Sevilla reclamó ante los Ministerios de Gobernación y Fomento contra la demolición del Convento de la Merced y otros monumentos.

Las pinturas provenías de los Conventos de Capuchinos suprimidos en 1835.

13. Ce chef-d'oeuvre occupait la place d'honneur au Musée du Louvre, à Paris, jusqu'an moment au il fut restitué a l'Espagne. [Nota en Catálogo de Laurent]

14. En el Museo Provincial de Córdoba.

15. Considerado como de Velázquez, en el Catálogo de 1867 figuraba como obra de Zurbarán.

16. Op. Cit. Suplemento al Catálogo....

17. Medida del cuadro que aparece en las fichas el Fichero Original Ruiz Vernacci.

18. Pasado al $M^{\circ}$ del Prado n² 2549 Fot: 22-1-1936.

19. En el Fichero Antiguo, dice : "entregado el negativo" [a la persona que lo encargara, por tanto no existe en el Archivo. 